\title{
Numerical simulation study on effectiveness of shielding structure on ultrasonic transmission tomography
}

\author{
Nan $\mathrm{Li}^{1^{*}} \mathbb{D}$, Kun $\mathrm{Xu}{ }^{2}$ and Sihai $\mathrm{Li}^{1}$
}

\begin{abstract}
Ultrasonic tomography (UT) is a non-invasive multiphase flow detection technique. In ultrasonic transmission tomography, reconstruction images are derived from a direct wave signal transmitted through the materials in the sensing area. The maximum values of the received direct wave from the reference pattern and the measured pattern are normally used to reconstruct the images. The speed of sound in the pipe wall and the materials in the sensing area affect the received signal. This in turn affects image quality-especially when the arrival time of the direct wave propagated through the sensing area is close to the arrival time of the direct wave propagated through the pipe wall. Here, we describe a novel shielding structure for ultrasonic transmission tomography. The relative variation ratio $(R V R)$ is defined and used to evaluate the validity of the shielding structure. Larger RVR values imply better signal resolution and better signal suppression. A simplified mode is discussed including the influence of the shielding structure's design parameters on the received direct wave. The optimized shielding structure size was determined. A comparison of the results between the normal pipe and the shielding pipe indicated that the RVR increased from 57.25 to $91.27 \%$. This proves that the shielding structure can suppress the effect of the signal from the pipe wall on received direct wave signals.
\end{abstract}

Keywords: Ultrasonic tomography, Transmission mode, Shielding, Direct wave, Suppression

\section{Introduction}

Ultrasonic waves are sensitive to an object's density and elasticity and can recover the component's distribution in the sensing area via measurement of signal at the ultrasonic transducers. This is known as ultrasonic tomography [1]. Similar to electrical capacitance/resistance tomography (ECT/ERT) [2-4], this technique is used in many industrial applications $[5,6]$. Researches have mainly focused on three parts. The first is ultrasonic tomography (UT) system design and sensor optimization. Since 1996, a series of related studies on UT system design and sensor optimization have been performed by Hoyle, $\mathrm{Li}$, and Schlaberg et al. [7-9]. Takahashi et al. designed a 2-MHz PVDF sensor for UT systems, and the sensor can be used to simultaneously monitor the changes in amplitude and phase [10].

\footnotetext{
* Correspondence: nan.li@hotmail.co.uk

${ }^{1}$ School of Automation, Northwestern Polytechnical University, Xi'an 710072, China

Full list of author information is available at the end of the article
}

The second is the image reconstruction algorithm. Rahiman et al. presented an HBR (hybrid-binary reconstruction) algorithm to reconstruct two-phase flow. The reconstruction speed is 10 frames/s [11]. In 2012, he presented a new method to improve the spatial resolution of the reconstructed images. The experimental results proved that this method is feasible and valid [12]. In 2014 and 2015, Yang et al. used ellipse and hyperbola algorithms to reconstruct a series of a priori circle targets based on the ultrasonic reflection tomography system $[13,14]$.

The third area of research is industrial applications. Brown et al. developed a UT system with applications in pneumatic conveyance [15]. Xu et al. demonstrated a UT system that monitors gas/liquid flow [16]. Schlaberg et al. built a 16-transducer ultrasonic reflection tomography systems for hydrocyclones, and the size and position of the air core could be monitored in real time. This system improved the separation efficiency of the hydrocyclone particles and slurries [17]. 
Transmission and reflection sensing modes are usually applied in the UT system. Attenuation of ultrasonic propagation is a common issue for all UT systems-especially during transmission mode [18]. Transmission mode uses a direct wave to determine whether there are any objects by measuring the shortest transmission time [19]. The situation becomes worse when the transducers are placed on the outer pipe wall.

The influence of the pipe's material on ultrasound attenuation is also significant. The received signals are more or less interfered by the ultrasound waves propagated from the pipe wall-especially when the speed of sound in the pipe wall is greater than the direct wave propagated across the sensing area. The usual way to solve this problem is to place the transducers in the pipe and make full contact with the internal media so that the influence of the pipe can be eliminated. However, these arrangements are not allowed in some cases. Therefore, a method to suppress the effect of the pipe on the received signal is necessary.

This paper describes techniques to suppress the influence of the signals propagating in the pipe wall on the direct waves received by transducers. First, a UT system with a transmission mode is introduced followed by a novel structure named the "shielding structure" in Section 2. The size of the shielding structure was optimized, and the effectiveness of the structure was evaluated. We compared the signals between the UT system with a shielding structure and without a shielding structure.

\section{Principle and methodology}

\subsection{Ultrasonic tomography}

A typical UT system consists of a data acquisition unit, a data processing unit, and an image reconstruction algorithm [20]. Transducers are evenly placed around a pipe wall; the number varies based on application. All transducers act as transmitters and receivers. For a system with $n$ transducers, there are $n(n-1) / 2$ measurements.

However, upon considering the quantity and quality of the data for better imaging, angular width $\theta$ of the main lobe of the transducer should be considered regardless of whether the system works in transmission mode or reflection mode. Five opposite transducers in a fan-shaped angle range are in the receiving state for an 8-transducer UT transducer (T1-T8) with $\theta=90^{\circ}$ (T1 is the transmitting transducer; Fig. 1). Each transducer is excited in turn, and 20 sets of data are obtained for imaging. Note that $\theta$ is a constant value when the design parameters of the transducers are determined. Normally it is less than $90^{\circ}$ (near $45^{\circ}$ ).

The sensitivity distribution at the sensing field was heterogeneous due to the nonlinearity of the measurement system. Three basic parameters must be considered before

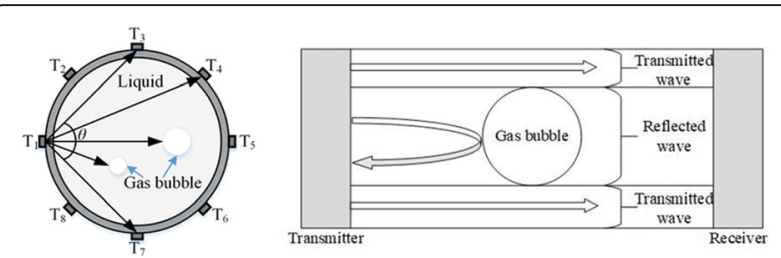

Fig. 1 A physical model and working principle of ultrasonic transmission tomography

measurement of the UT sensor: the speed $(c)$, wavelength $(\lambda)$, and frequency $(f)$ of the ultrasonic wave. The parameters satisfy the following function: $\lambda=c / f$.

The selection of transducer frequency thus becomes an important part. To detect the object in the sensing area, the smallest size of object $a$ and excitation frequency $f$ should satisfy Eq. (1), and the diffraction can be ignored [21].

$$
\frac{2 \pi f a}{c} \gg 1
$$

For ultrasonic transmission tomography, only direct signals received by the transducers will be applied to determine whether there is any object by measuring the time of flight (TOF; Fig. 2). When T1 is set as an ultrasound transmitter, the received signal of R1 consists of the signal propagated from a directly transmitted path, a reflecting path from the bubble and the inner pipe wall, and an arc path in the pipe wall. The LBP (linear back projection) algorithm is usually used in ultrasonic transmission tomography. This algorithm mainly relies on the maximum amplitude value in direct wave time range.

The signal propagated in the pipe wall provides no information on gas bubble detection because the ultrasound waves in the pipe wall do not contact the bubble. This part of the signal could be treated as "noise" for imaging. It is easy to determine the range A signal by calculating the TOF, which is the arrival time of the direct wave (Fig. 2b). However, this time period not only includes direct waves from path 1 but also the ultrasonic wave signal propagated from paths 2 and 3 in some cases. It is easy to reconstruct images when the arrival time of the direct wave is shorter than the time of the reflection signal by the pipe wall and gas bubble. Of course, it is shorter than the time that the wave propagates through the pipe wall. However, in most of the cases, the arrival times of the directly transmitted signals in water may be close to or later than that in the pipe wall with specific excitation frequency or for some special materials in the pipe wall. This could be a problem for signal analysis, and it will affect the quality and accuracy of the reconstructed images. 

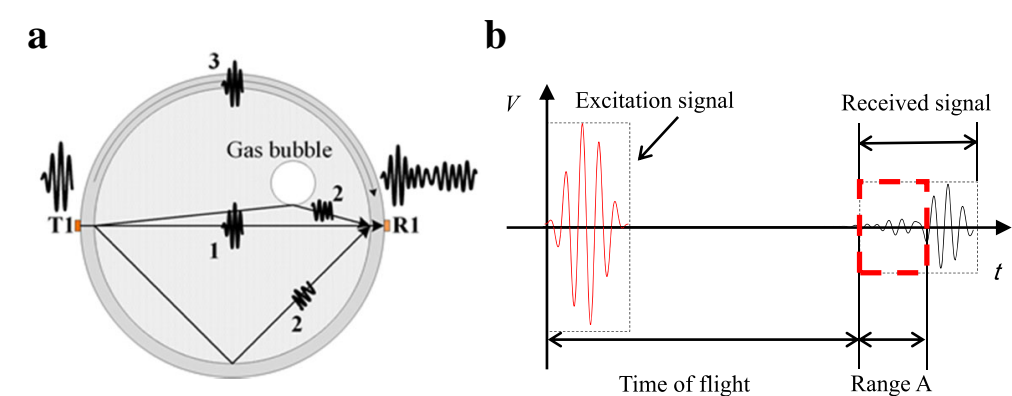

Fig. 2 a The three kinds of paths for ultrasonic wave propagation excitation signal and received signal (note: 1 refers to direct wave signal, 2 is reflected signal from bubble and pipe wall, and 3 is the signal propagated through the pipe wall). $\mathbf{b}$ Measurement principle of ultrasonic transmission mode

In theory, the receiver cannot receive a direct wave signal when the gas bubble is placed on the propagation path between the transmitter and the receiver. Thus, the presence of the gas bubble can be identified. However, the signal propagated in the pipe wall also can be received at that time, and it certainly will affect the signal analysis. Therefore, a shielding structure is necessary.

\subsection{Shielding structure}

The shielding structure is intended to suppress the effect of the signals that propagate into the pipe wall. $\mathrm{T} 1$ is a transmitter, while R1 to R3 are receivers in Fig. 3. The shielding bulges are arranged on the outer of the pipe wall between a transmitter and a receiver. The ultrasound wave propagated into the pipe wall will be "absorbed" by these bulges.

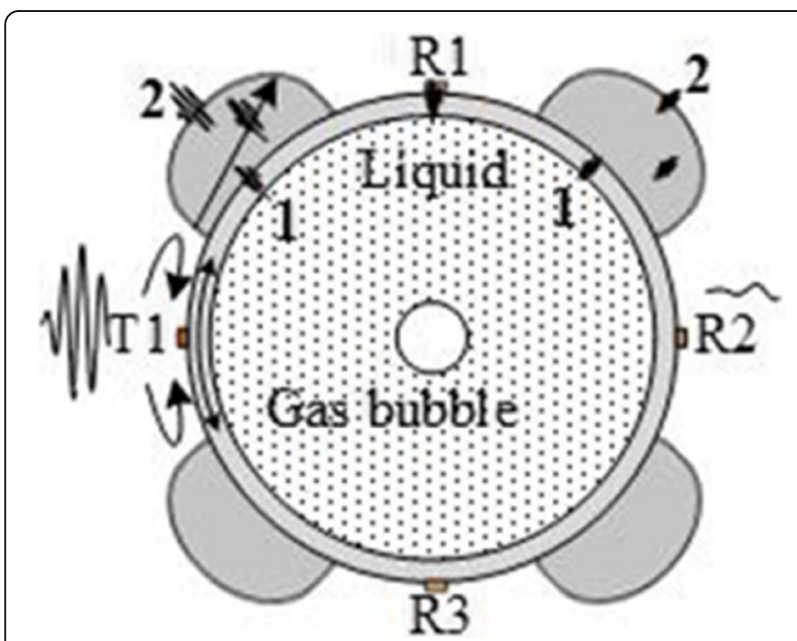

Fig. 3 A physical model and working principle of shielding structure in ultrasonic transmission tomography. (Note: 1 refers to ultrasonic waves propagated in pipe wall and 2 is an ultrasonic wave propagated in bulges)

\section{Modeling and setups}

To simplify the model, two opposing transducers are arranged outside of the pipe wall along the horizontal direction of the sensing section. One transducer is transmitter and another is receiver. The point source is excited by a sine wave signal modulated by a 5 -cycle Hanning window. The excitation is $14 \mathrm{~Pa}$, and the center frequency is $300 \mathrm{kHz}$. The pipe is made of acrylic with an outer diameter of $110 \mathrm{~mm}$; the thickness of the pipe wall is $5 \mathrm{~mm}$. A gas bubble is located at the center of the sensing area with a diameter of $20 \mathrm{~mm}$.

Next, a parametric study was applied to evaluate the optimal size of the shielding structure. Two acrylic bulges with an initial height $h$ of $10 \mathrm{~mm}$ and initial width $w$ of $10 \mathrm{~mm}$ were symmetrically placed outside the pipe wall-these are perpendicular to the horizontal direction. The range of bulges $h$ and $w$ vary from 10 to $40 \mathrm{~mm}$, respectively. The diagram of the model is shown in Fig. 4a, and the sound field distributions of 50, 70, and $80 \mu$ s are illustrated in Fig. 4 from (b) to (d). Note that the speed of the ultrasonic wave in acrylic and water are 2730 and $1473 \mathrm{~m} / \mathrm{s}$, respectively. This means that the arrival time of the ultrasonic wave propagated though the pipe wall is close to a direct wave transmitted through the water.

The gas bubble will block direct wave propagated across sensing section. The effect of the size and location of the gas bubble on the direct ultrasound wave signals needs to be evaluated. The bubble position moves on the connecting line of the two transducers ( $\mathrm{T} 1$ and R1) to guarantee the direct wave is blocked, i.e., the $x$ axis direction. Here, two situations are considered: (1) The bubble diameter $d$ changes from 20 to $60 \mathrm{~mm}$ with a $20 \mathrm{~mm}$ interval at the center of the Section 2). The center position of the bubble moves from the position $(-30,0)$ to $(30,0)$ with a $(10,0)$ interval where the position $(0,0)$ is the center of sensing section. The results are shown in Fig. 5. Considering differences in the sound speed in the pipe and water, the theoretical time range of the received direct wave is 71.6 to $88.2 \mu \mathrm{s}$. 

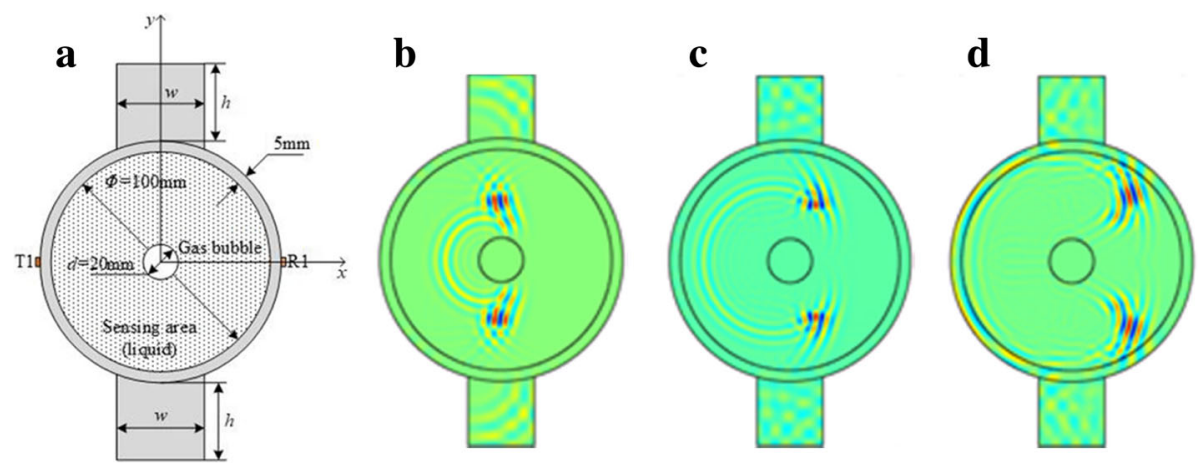

Fig. 4 A physical model and propagation process of ultrasonic wave inside the bulges: a physical model b $50 \mu s$, c $70 \mu s$, and $\mathbf{d} 80 \mu s$

The principles of the imaging algorithm suggest that we only care about the maximum value of the received direct wave signal. The results show that the maximum value of received signal is slightly affected by changes in the diameters and positions of the gas bubble. Therefore, the influence of $d$ and $x$ on the received signal is ignored in this follow-up study.

\section{Results and discussion}

Equation (1) shows that the diffraction can be ignored in the following ultrasonic transmission tomography experiments. The validity of the shielding structure is evaluated in two steps based on the model demonstrated in Fig. 4a.

Step 1: The received signals of $\mathrm{R} 1$ on the pipe without a shielding structure will be measured and analyzed for two situations: (a) the pipe is full of water or (b) a single gas bubble is located at the center state. In a follow-up study (normal pipe condition, NPC), these received values will be used as reference signals.

Step 2: The received signals of $\mathrm{R} 1$ on the pipe without a shielding structure will be measured and analyzed for two situations: (a) the pipe is full of water and (b) a single gas bubble located at the center state. In a follow-up study (shielding pipe condition, SPC), these received values are used to compare with the reference signals and to then reconstruct images.

At the same time, a set of experiments is given as a comparison. In the experiment, the transducer has dual functions that it can be used either as a transmitter or as a receiver. The shape of piezoelectric transducer is cylinder. Its diameter and thickness are 10 and $6.7 \mathrm{~mm}$, respectively. The frequency of $300 \mathrm{kHz}$ is a center frequency of thickness vibration direction.

The reference signals from simulated results and experimental results are shown in Fig. 6. Note that only direct wave signals are given in follow-up figures, i.e., the range of $t$ is from 70 to $88.5 \mu$ s. Here, NPC-FW denotes that the model is full of water in NPC; NPC-SB refers to the model in which a single bubble is placed at the center of the NPC. Two maximum points are highlighted in Fig. 6 due to the image reconstruction algorithm. The relative variation ratio, $R V R$, is defined as the following equation to evaluate the effectiveness of the shielding structure.
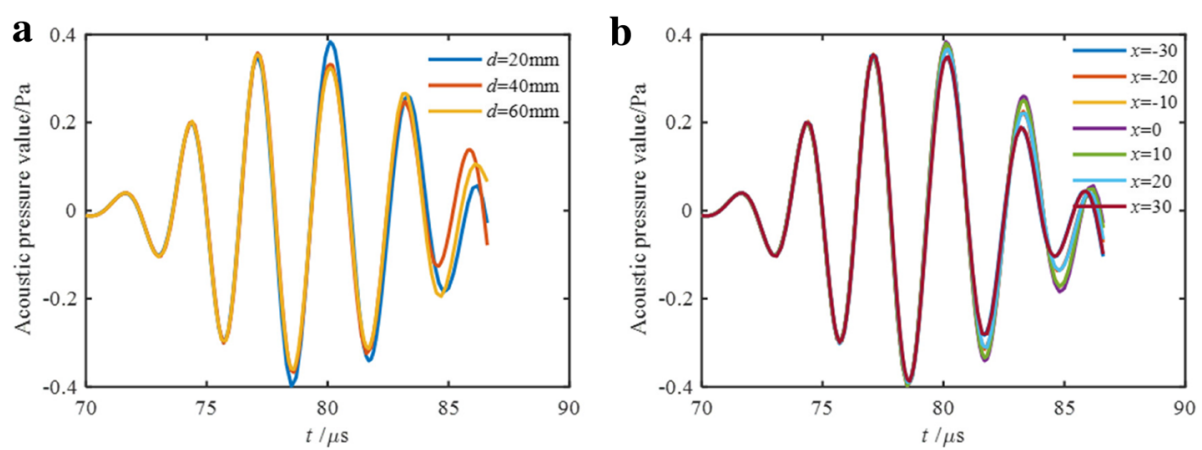

Fig. 5 The effects of the size and location of the gas bubble on received signal of direct ultrasound waves: a for different $d$ of the gas bubble at the position $(0,0)$ and $\mathbf{b}$ for different positions of gas bubble from $(-30,0)$ to $(30,0)$ when $d$ is $20 \mathrm{~mm}$ 

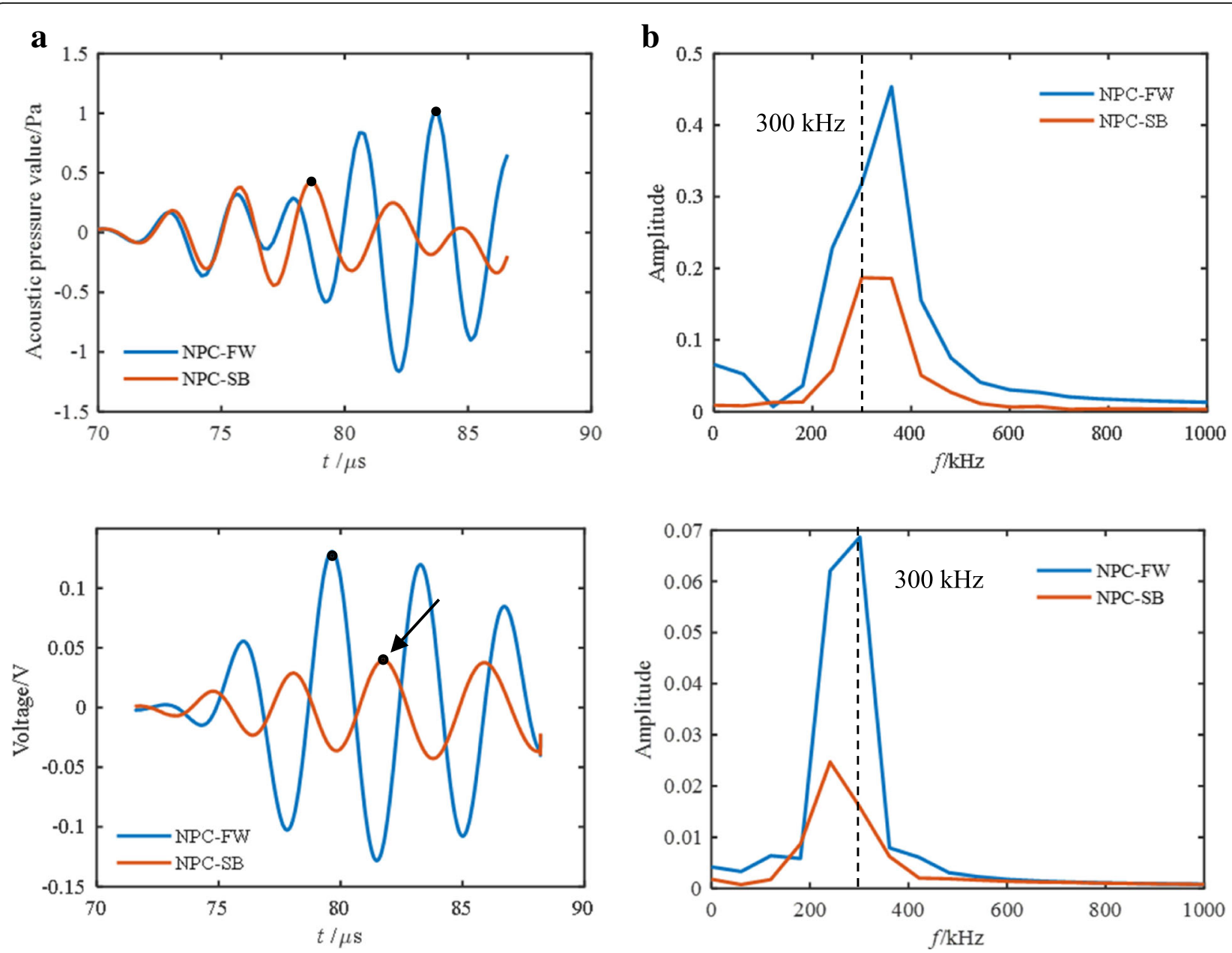

Fig. 6 A comparison of the received direct signal of ultrasound waves between NPC-FW and NPC-SB: a time domain signals of simulated and experimental results and $\mathbf{b}$ frequency domain signals of simulated and experimental results

$$
R V R=\frac{M_{\max }-M_{\max }^{\prime}}{M_{\max }} \times 100 \%
$$

Here, $M_{\max }$ is received maximum value of direct wave signal in the pipe without gas bubbles. $M^{\prime}{ }_{\max }$ is received maximum value of direct wave signal in the pipe with a single bubble. $R V R$ plays a key role in imaging part-larger $R V R$ values imply better imaging quality [20,21].

The $M_{\max }$ is $(83.7,1.001)$ for NPC; $M_{\text {max }}^{\prime}$ is $(78.6,0$. 4279). The $R V R$ are 57.25 and $69.53 \%$ corresponding to the simulated and experimental results, respectively (Fig. 6). The spectrum domain signals of the simulated and experimental results show that the most of the energy is concentrated around $300 \mathrm{kHz}$. These results indicate that the results correspond with the simulation. The numerical approach can provide an economical way to tackle the variables, and $2 \mathrm{D}$ approximations also support quick simulations. The following study will concentrate on the simulation.
For SPC, the proper size of the acrylic bulges should be decided before the $R V R$ can be calculated. These conditions are similar to Section 3. The effects of size and location of the gas bubble on the direct wave can be ignored. Thus, we set a single gas bubble at the center position of the sensing area with a $20 \mathrm{~mm}$ diameter. The maximum values of the measurement direct wave with a different combination of $w$ and $h$ are listed in Table 1.

Table 1 The maximum acoustic pressure value of the direct waves with different width $w$ and height $h$ of shielding structure $(\mathrm{Pa})$

\begin{tabular}{lllll}
\hline$h / \mathrm{mm}$ & $w / \mathrm{mm}$ & & & \\
\cline { 2 - 5 } & 10 & 20 & 30 & 40 \\
\hline 10 & 0.2157 & 0.1414 & 0.2485 & 0.2599 \\
20 & 0.1846 & 0.1162 & 0.1538 & 0.2525 \\
30 & 0.1727 & 0.1023 & 0.0916 & 0.1515 \\
40 & 0.1848 & 0.1174 & 0.1156 & 0.0974 \\
\hline
\end{tabular}



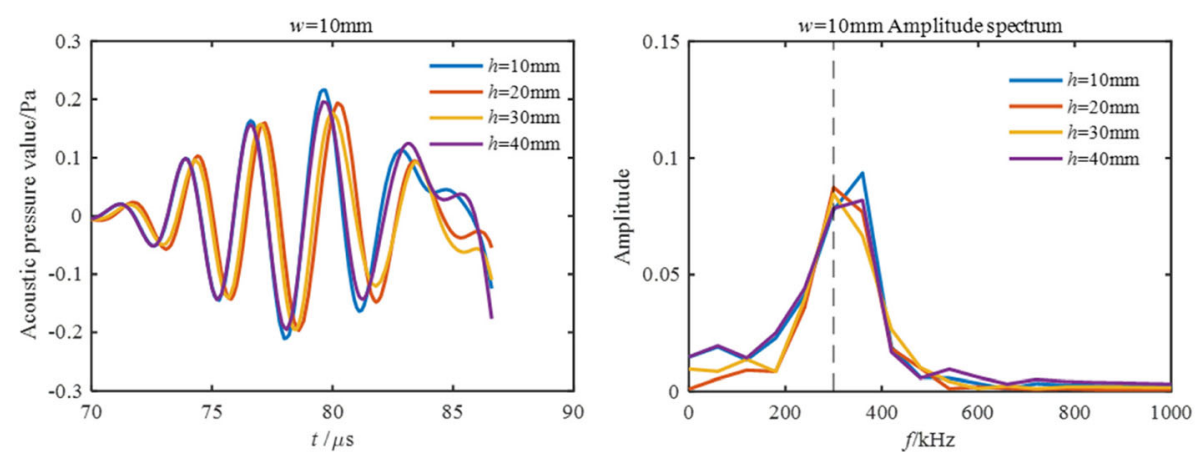

(a1)

(a2)
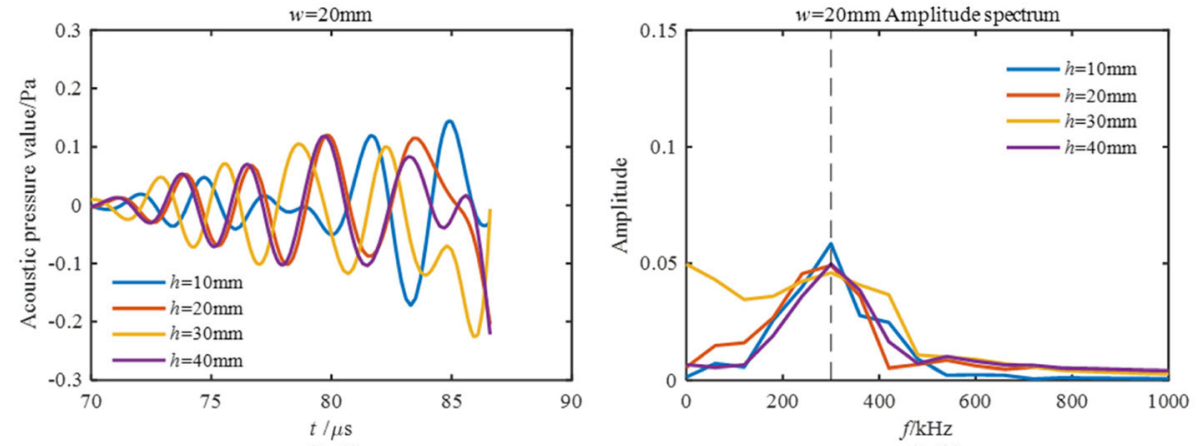

(b1)

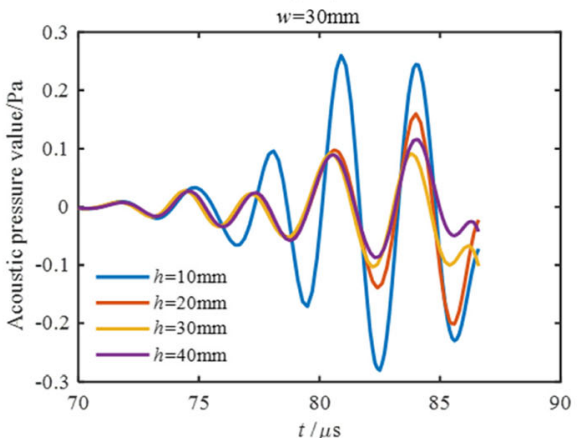

(b2)

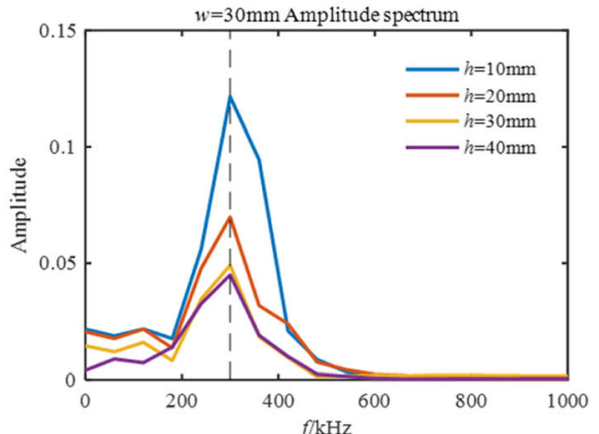

(c1)

(c2)

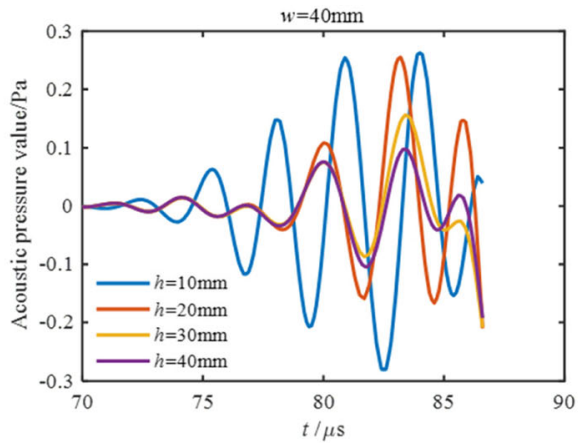

(d1)

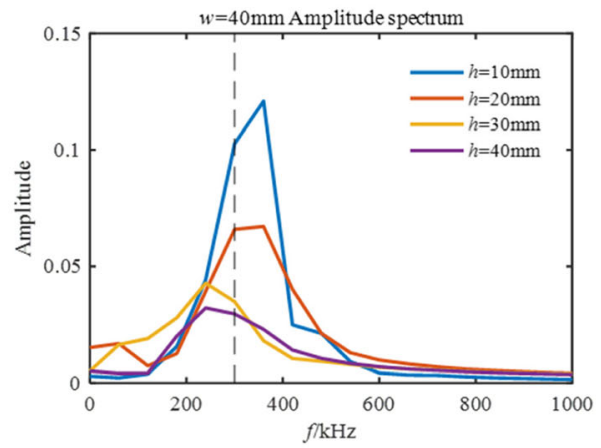

(d2)

Fig. 7 The time domain and frequency domain signal of received direct waves with different $w$ and $h$ of shielding structures: a $w=10 \mathrm{~mm}$ (a1) time domain, (a2) frequency domain, b $w=20 \mathrm{~mm}$ (b1) time domain, (b2) frequency domain, $\mathbf{c} w=30 \mathrm{~mm}$ (c1) time domain, (c2) frequency domain, and $\mathbf{d} w=40 \mathrm{~mm}(\mathrm{~d} 1)$ time domain, (d2) frequency domain 

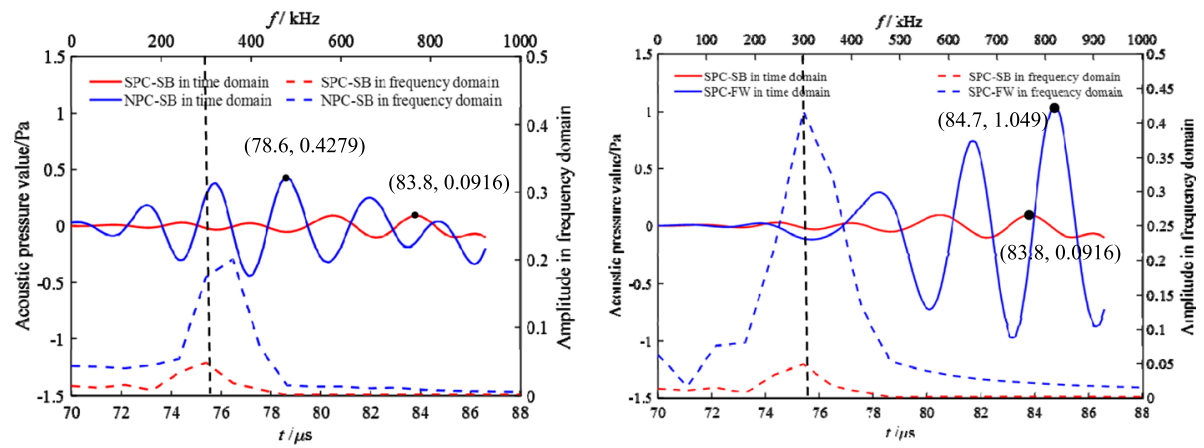

Fig. 8 The comparison of received direct signal of ultrasound waves from time domain and frequency domain: a SPC-SB and NPC-SB and b SPC-SB and SPC-FW

Both $w$ and $h$ can affect the maximum value of direct wave measurements; there is no monotonic changing trend. For $h>10 \mathrm{~mm}$, when $w$ equals to $h$, the maximum acoustic pressure value of the direct wave is the smallest-these are highlighted by the red box in Table 1. The smallest value among all combinations $(0.0916 \mathrm{~Pa})$ is derived when $w$ and $h$ are $30 \mathrm{~mm}$. It is difficult to have more definite conclusions from only Table 1. Therefore, the time domain and frequency domain signal of the received direct waves are presented in Fig. 7.

Figure 7 suggests that the time domain signals have no common change trend with changes in $w$. At $w=10 \mathrm{~mm}$, the waveform is slightly changed, and there are small changes in the phase and amplitude (Fig. 7(a1)). This point also can be observed in the frequency domain shown in Fig. 7(a2). Note that for $h$ values equal to 10 and $40 \mathrm{~mm}$, the highest amplitude is at $380 \mathrm{kHz}$, but the excitation is $300 \mathrm{kHz}$. This means that the shielding structure does not filter out the influence of the signal propagated through the pipe wall. Therefore, this size is not suitable for the shielding structure of this study.

At $w=20 \mathrm{~mm}$, the main difference of the waveform is in the phase change (Fig. 7(b1)). The frequency domain signal analysis shows that the peak value appears at $300 \mathrm{kHz}$ for all of $h$ except $h=30 \mathrm{~mm}$ (Fig. 7(b2)). The maximum value is insensitive to height of the shielding structure. This is not the best choice for shielding structures. At $w=30 \mathrm{~mm}$, the phase shift is small (Fig. 7(c1)), and it also has a single peak at $300 \mathrm{kHz}$ in the frequency domain (Fig. 7c2). Most of the energy is concentrated at $300 \mathrm{kHz}$ for all $h$ values compared with the frequency domain signals when $w=20 \mathrm{~mm}$ and $w$ $=40 \mathrm{~mm}$. When $w=40 \mathrm{~mm}$, there is a large phase shift when $h$ increases from 10 to $20 \mathrm{~mm}$ (Fig. 7(d1)). The phase shift becomes smaller when $h$ is larger than $20 \mathrm{~mm}$. The frequency domain signals indicate that the performance of the shielding structure at a $40-\mathrm{mm}$ width is better than the shielding structure with $20 \mathrm{~mm}$ (Fig. $7(\mathrm{~d} 2)$ ), but this is worse than the structure with $30 \mathrm{~mm}$. Therefore, both the $w$ and $h$ of the shielding structure should be $30 \mathrm{~mm}$ for optimal performance. To evaluate the effectiveness of the shielding structure and compare the received direct signal of the ultrasound waves between SPC-SB and NPC-SB, the SPC-WF and SPC-SB are shown in Fig. 8 when $w$ and $h$ of the shielding structure are $30 \mathrm{~mm}$.

The maximum value of SPC-SB is smaller than the NPC-SB in both the time domain and the frequency domain. The peak value of NPC-SB in the frequency domain is $350 \mathrm{kHz}$. The peak values of SPC-SB and SPC-FW are $300 \mathrm{kHz}$. This means that the signals propagated through pipe wall have been suppressed. After calculation, the SPC $R V R$ is $91.27 \%$. This is obviously greater than RVR (57.25\%) of NPC. These results can prove that the "shielding structure" suppresses the useless ultrasonic waves propagated in the pipe wall.

\section{Conclusions}

The simulated and experimental results suggest the following:

(i) A parametric study determined the optimal size of the shielding structure. The "shielding structure" can effectively suppress the signal propagated in pipe wall. Simulation analysis shows that the proper size of acrylic bulges should be $30 \mathrm{~mm}$ for $w$ and $h$. In this case, the amplitude of the received ultrasonic direct waves of SPC-SB is much smaller than NPC-SB.

(ii) Greater $R V R$ values result in better imaging quality. The $R V R$ of SPC is $91.27 \%$, which is obviously greater than the $R V R$ of NPC.

(iii) The spectrum analysis shows that the central frequency of SPC-SB and SPC-FW signal is $300 \mathrm{kHz}$ when $w$ and $h$ are $30 \mathrm{~mm}$. This suggests that the received direct wave signal is only from the water, and the influence of the signals from the pipe wall is suppressed.

\section{Abbreviations}

ECT/ERT: Electrical capacitance/resistance tomography; HBR: Hybrid-binary reconstruction; LBP: Linear back projection; NPC: Normal pipe condition; 
NPC-FW: Model is full of water in NPC; NPC-SB: Model in which a single bubble is placed at the center of the NPC; RVR: Relative variation ratio; SPC: Shielding pipe condition; TOF: Time of flight; UT: Ultrasonic tomography

\section{Funding}

This work was financially supported by the Project 51475013, 51105008 of National Natural Science Foundation of China and the Fundamental Research Funds for the Central Universities (31020170QD028).

\section{About the authors}

Nan Li received his B.S. and Ph.D. degree in 2004 and 2010, respectively, from Xidian University. He has worked as an associate professor in Beijing University of Technology from 2010 to 2016. Currently, he is an associate professor in Northwestern Polytechnical University. His main research interests include non-destructive testing and evaluation, automatic control, and process tomography.

Kun Xu received his B.S. degree in 2014 from Baicheng Normal University. He is currently a master student at Beijing University of Technology. His main research interests include non-destructive testing and evaluation, process tomography, and sensor technology.

Sihai Li received the M.S. and Ph.D. degrees in Navigation Guidance and Control from the School of Automation, Northwestern Polytechnical University (NWPU), Xi'an, China, in 1988 and 2000, respectively. He has been a professor of Northwestern Polytechnical University since 2008. He is the director of INS and Multi-sensor Integrated Navigation Research Group at NWPU. He is a leading expert in the area of aviation inertial navigation and integrated navigation systems in China with over 25 years' industrial experience. He is currently a council member of Chinese Society of Inertial Technology (CSIT).

\section{Authors' contributions}

NL proposed the shielding structure. He also analyzed the data and signals. KX performed the simulations. NL, KX, and SL wrote the paper. All authors read and approved the final manuscript.

\section{Competing interests}

The authors declare that they have no competing interests.

\section{Publisher's Note}

Springer Nature remains neutral with regard to jurisdictional claims in published maps and institutional affiliations.

\section{Author details}

'School of Automation, Northwestern Polytechnical University, Xi'an 710072 , China. ${ }^{2}$ College of Mechanical Engineering and Applied Electronics Technology, Beijing University of Technology, Beijing 100124, China.

Received: 27 January 2018 Accepted: 11 April 2018

Published online: 23 April 2018

\section{References}

1. Schlaberg HI, Yang M, Hoyle BS. Ultrasound reflection tomography for industrial processes. Ultrasonics. 36(1), 297-303 (1998)

2. M Mao, J Ye, H Wang, et al., Evaluation of excitation strategies for $3 D E C T$ in gas-solids flow measurement. IEEE Sensors J. 16(23), 8523-8530 (2016)

3. N Li, M Cao, K Liu, et al., A boundary detecting method for post-tensioned pre-stressed ducts based on Q-factor analysis. Sens. Actuators A Phys. 248, 88-93 (2016)

4. A Lehikoinen, JMJ Huttunen, S Finsterle, et al., Dynamic inversion for hydrological process monitoring with electrical resistance tomography under model uncertainties. Water Resour. Res. 46, W04513 (2010)

5. XX Dong, C Tan, Y Yuan, et al., Measuring oil-water two-phase flow velocity with continuous-wave ultrasound Doppler sensor and drift-flux model. IEEE Trans. Instrum. Meas. 65(5), 1098-1107 (2016)

6. DL Núñez, MA Molero-Armenta, MAG Izquierdo, et al., Ultrasound transmission tomography for detecting and measuring cylindrical objects embedded in concrete. Sensors 17(5), 1085 (2017)

7. BS Hoyle, Process tomography using ultrasonic sensors. Meas. Sci. Technol. 7, 272-280 (1996)

8. W Li, BS Hoyle, Ultrasonic process tomography using multiple active sensors for maximum real-time performance. Chem. Eng. Sci. 52(13), 2161-2170 (1997)
9. HI Schlaberg, M Yang, BS Hoyle, et al., Wide-angle transducers for realtime ultrasonic process tomography imaging applications. Ultrasonics 35, 213-221 (1997)

10. S Takahashi, H Ohigashi, Ultrasonic imaging using air-coupled P (VDF/TrFE) transducers at 2 MHz. Ultrasonics 49, 495-498 (2009)

11. MHF Rahiman, RA Rahim, M Tajjudin, Ultrasonic transmission-mode tomography imaging for liquid/gas two-phase flow. IEEE Sensors J. 6(6), 1706-1715 (2006)

12. MHF Rahiman, RA Rahim, HA Rahim, et al., Novel adjacent criterion method for improving ultrasonic imaging spatial resolution. IEEE Sensors J. 12(6), 1746-1747 (2012)

13. L Yang, $C X u, X$ Guo, Ring-shaped array ultrasound imaging using ellipse algorithm. Comput. Model. New Technol. 18(12D), 315-320 (2014)

14. L Yang, C Xu, X Guo, et al., Ultrasonic tomography of immersion circular array by hyperbola algorithm. Telkomnika 13(1), 65-75 (2015)

15. GJ Brown, D Reilly, D Mills, Development of an ultrasonic tomography system for application in pneumatic conveying. Meas. Sci. Technol. 7, 396-405 (1996)

16. LJ Xu, Y Han, LA Xu, et al., Application of ultrasonic tomography to monitoring gas/liquid flow. Chem. Eng. Sci. 52(13), 2171-2183 (1997)

17. HI Schlaberg, FJW Podd, BS Hoyle, Ultrasound process tomography system for hydrocyclones. Ultrasonics 38, 813-816 (2000)

18. Q Su, C Tan, F Dong, Mechanism modeling for phase fraction measurement with ultrasound attenuation in oil-water two-phase flow. Meas. Sci. Technol. 28(3), 035304 (2017)

19. G Steiner, C Deinhammer, Ultrasonic time-of-flight techniques for monitoring multi-component processes. Elektrotechnik Informationstechnik 126(5), 200-205 (2009)

20. LJ Xu, LA Xu, Ultrasound tomography system used for monitoring bubbly gas/liquid two-phase flow. IEEE Trans. Ultrason. Ferroelectr. Freq. Control 44(1), 67-76 (1997)

21. $\amalg J \mathrm{Xu}, \mathrm{LA} \mathrm{Xu}, \mathrm{Gas} /$ liquid two-phase flow regime identification by ultrasonic tomography. Flow Meas. Instrum. 8(3/4), 145-155 (1988)

\section{Submit your manuscript to a SpringerOpen ${ }^{\circ}$ journal and benefit from:}

- Convenient online submission

- Rigorous peer review

- Open access: articles freely available online

- High visibility within the field

- Retaining the copyright to your article

Submit your next manuscript at $>$ springeropen.com 